\title{
Lecture-experiment to demonstrate the excitation of electricity by light
}

\section{Wilhelm Hallwachs}

To cite this article: Wilhelm Hallwachs (1890) Lecture-experiment to demonstrate the excitation of electricity by light, Philosophical Magazine Series 5, 30:182, 127-128, DOI: $10.1080 / 14786449008619996$

To link to this article: http://dx.doi.org/10.1080/14786449008619996

曲 Published online: 08 May 2009.

Submit your article to this journal $[\pi$

Џ Article views: 3

Q View related articles $\asymp$ 
seems well suited for accurate measurements of the wave-length and of the decrement of the vibrations.-Wiedemann's Annalen, xl. p. $399(1890)$.

\section{LECTURE-EXPERIMENT TO DEMONSTRATE THE EXCITATION OF ELECTRICITY BY LIGHT, BY WILHELM HALLWACHS.}

In the first experiments on the excitation of electricity by light, only very feeble positive charges were obtained of the magnitude of a volt. MM. Bichat and Blondlot then found a method of increasing the charges by directing a current of air against the irradiated plate by which they obtained increases of potential up to 30 volts. This method of increasing the excitation afforded at the same time an insight into the connexion between the excitation of electricity by light and the discharge of electricity by light, which latter was increased by the electrostatic power of the negative charges imparted to the plates, just as by a current of air. This connexion was made clear by the experiments of Prof. Righi, who demonstrated the proportionality between the potential produced by radiation and the electrostatic forces which occur at the surface. In repeating the experinents of Bichat and Blondlot, I have obtained charges up to 100 volts by blowing against the bottom of a brass vessel suspended in a sheath.

In order to show in a lecture the excitation of electricity by light we take advantage of the current of air ; for then, if the field of observation is sufficient and the lamp does not burn too long, we may dispense with the troublesome screens against induction. By means of the following arrangement I have been able to show the phenomenon to a large circle of hearers. In the luminous cone of the electric light, after removing any glass lenses in the lantern, a freshly polished zinc plate fixed to a shellac support is introduced laterally. The rays of light which pass by on the side strike a lens at some distance, which concentrates them on the gold leaf of a. Hankel's electrometer. After removing the eyepiece, the object-glass of the microscope of the electrometer projects an image of the gold leaves on a screen, on which a scale is affixed to observe the risplacement of the gold leaves. When the electrometer was charged with 20 chromic-acid elements and when the plates of the electrometer were tolerably near, the desired sensitiveness was obtained. In order to measure the latter a rigid wire was placed on the electrometer-battery, a few elements removed from the point put to earth, and connected by the conductor with the gold leaf.

In order to make the experiment, the gold leaf and the zinc plate are connected with each other, a disk of mica is introduced in the path of the rays coming from the lamp, the zinc plate is put to earth for a moment, and after removing the mica disk no perceptible change is observed in the adjustment of the gold leaves if the sensitiveness of the electrometer is not exceptionally great. If 
there is no deflexion, it shows that the space in which the observations are made is free from the products of combustion of the lamp. When the mica disk has been again pushed forward the zinc plate is blown on from a hand-bellows, when there is no defiexion of the gold leaf. If then the mica disk is withdrawn, a few separate puffs with the bellows are sufficient to produce considerable deflexions, so as to drive the leaf off the scale. That the direction of the deflexion corresponds to a positive electrification follows from the sign of the potential when the electrometer is charged for determining its sensitiveness, and from the deflexions thereby produced. Wiedemann's Annalen, xl. p. 343 (1890).

\section{INVESTIGATION OF ELECTRICAL RESONANCE. BY DR. ERNEST LECHNER.}

The author describes first of all a method of observing electrical vibrations in wires. Opposite each of the two terminal plates of a Hertz's arrangement for vibrations an equally large insulated plate is arranged, and from each plate is led a wire several metres in length, the two wires being parallel. Over the end of the wire a rarefied tube, without an electrode, is placed, which becomes luminous in consequence of the electrical oscillations in the wires. If the parallel wires are connected by a cross wire the luminosity in general ceases. If this bridge is moved backwards and forwards along the parallel wires, a few very sharply defined places are found where the tube suddenly becomes luminous; these are the ventral segments of the electrical motion.

After the author had studied the conditions of the experiment, and had shown that the whole is an electrical phenomenon of resonance, the shape and position of the electrical wave was precisely determined by seeking the common ventral segment. The influence of the introduction of capacities at the end of the wires was also examined.

The author invariably found that Hertz's observations were always confirmed; in one important point, however, he obtained a different result. While Hertz found for the velocity of electriuity in wires 200,000 kilometres per second, the author obtained within 2 per cent. of the exact velocity required by Maxwell's and all other theories; he cannot, however, explain why his results differ from that of Hertz. A possible source of error in the experiments of Hertz was found on closer inquiry to be too small. As the method used by the author is very simple and comprehensive, and can be very easily made, in the form even of a lecture-experiment, he considers his value not only theoretically but also practically the most probable.-Wiener Berichte, May 8, 1890. 